\title{
Thermodynamics of Thermoelectricity
}

\author{
Christophe Goupil \\ ENSICAEN, UMS-CNRT, CRISMAT
}

France

\section{Introduction}

Discovered at the beginning of the nineteen century, the thermoelectric effects nicely reflect the richness of the out of equilibrium thermodynamics of coupled phenomena. In 1821 by John Seebeck (Seebeck 1821), (Seebeck 1823), (Seebeck 1826) firstly observed the coupling of two potentials, the electrochemical potential and the temperature. In 1834 Jean Peltier (Peltier 1834) demonstrated that heat flux and electrical current could be coupled. Then in 1855, W. Thompson, future Lord Kelvin, using thermodynamic arguments, discovered that the Seebeck and Peltier effects were in fact not independent (Thompson 1848), (Thompson 1849), (Thompson 1852), (Thompson 1854), (Thompson 1856) giving decisive arguments in favor of a complete and compact description of all these phenomena. Only latter, in 1931, these coupled thermodynamic forces and fluxes where described in a very general form when Lars Onsager proposed a theoretical description of linear out of equilibrium thermodynamic processes. In two major articles the fundamentals of thermodynamics of dissipative transport were developed in a consistent way (Onsager 1931a), (Onsager 1931b). Next, In 1948, Callen developed the Onsager theory in the case of thermal and electrical coupled fluxes, leading to a coherent thermodynamical description of the thermoelectric processes(Callen 1948), (Domenicali 1954). Then, in the middle of the last century Abraham Ioffe, considering both thermodynamics and solid state approaches, extended the previous developments to the microscopic area, opening the door for material engineering and practical applications (Ioffe 1960). He introduced the so-called "figure of merit" ZT, which, as a material parameter, gather the different transport coefficients, leading to an efficient classification of the various thermoelectric materials. The contains of this chapter is divided in six sections. In a first section we remind the basic thermodynamics of thermoelectricity from classical thermodynamic cycle. The second section is devoted to the Onsager description of out equilibrium thermodynamics of coupled transport processes. In a third section the consequence of the Onsager theory are derived leading to the expressions of heat and entropy production. The fourth section is devoted to the presentation of the general conductance matrix. Using these latter the concept of relative current and thermoelectric potential are exposed in the fifth section.Then in the final section the traditional expressions of the efficiencies and Coefficients Of Performance (COP) are revisited using the thermoelectric potential approach.

\section{The thermoelectric engine}

In a first approach we propose here to consider the analogies between a classical steam engine, and a thermoelectric material (Vinning 1997). The principle analogy is the fact that, in both 
systems, the entropy is transported by a fluid, which, in the present case, is a gas of electron, also called "Fermi gas" since electrons are Fermions. At first this Fermi gas can be considered to be a perfect gas. Then the equivalent partial pressure of the fluid in the system is the electrochemical potential $\mu_{e}$.

$$
\mu_{e}=\mu_{c}+e V
$$

where $\mu_{c}$ is the chemical potential, $e$ the electron charge, and $V$ the electrical potential.

Then the "gas" equivalences for the steam and thermoelectric engines are,

\begin{tabular}{||lll||}
\hline \hline Molecular gas & $P$ & $T$ \\
Fermi gas & $\mu_{\ell}(T, r)$ & $T$ \\
\hline \hline
\end{tabular}

Then we can draw a picture of a schematic thermoelectric cell:

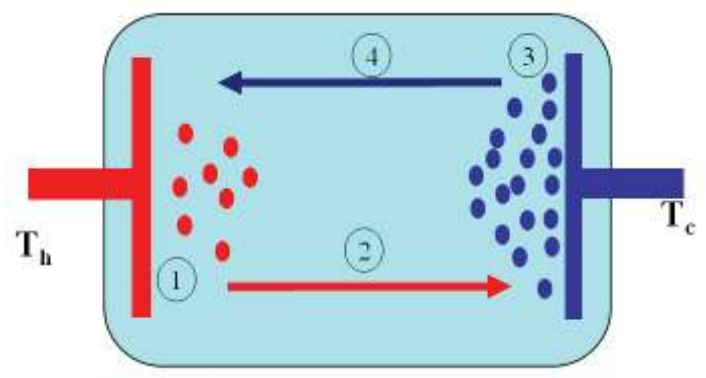

Fig. 1. Thermoelectric "Engine".

One end of the cell is maintained at the temperature $T_{h}$ and the other at temperature $T_{c}$ with $T_{h}>T_{c}$. From basic gas kinetic theory we know that we get large velocities and small gas density at the hot end and reduced velocities and large gas density at the cold end. It should be noticed here that, since heat flows from hot to cold end, the system cannot be considered under equilibrium condition meanwhile the average carrier flux is zero. We see that the gradient of carrier density is directly driven by the temperature difference. Since the carriers are charged particles, it produces an electrochemical difference, commonly called voltage difference, which is induced by the application of a temperature difference. This illustrates the coupling of the electrochemical potential gradient and the temperature gradient. Next, since the average carrier flux is zero, meanwhile heat is transported, we get the same values of local fluxes of carriers from hot to cold and cold to hot. From this observation we can conclude that heat and carrier fluxes are coupled. While very simple, this description contains the principal contributions to the thermoelectric processes ${ }^{1}$. The thermodynamical cycle is a Carnot cycle with two "'isothermal branches"' (1 and 3) and to "'adiabatic branches"' (2 and 4). We can then estimate the principle sources of entropy of the working system that are the non-isothermal heat transfers and the non-adiabatic travel of the carriers from cold to hot sources and hot to cold. The principle sources of entropy in the adiabatic branches are the collisions between carriers and the interactions of the carriers with the cristal lattice of the material. It can be noticed that the entropy is transported by the carriers of the Fermi gas. We

\footnotetext{
${ }^{1}$ In the case of uncharged particles we would also observed a similar process, called "Soret effect" with the diffusion of particles but, of course, no electrical drift contribution.
} 
will see latter that we can defined an "entropy per carrier" which is a fundamental parameter of the thermoelectric process. The principle weakness of this schematic presentation is the complete absence of a correct out of equilibrium thermodynamic in the description. Then we now need to define properly the conditions of this working system. This is the subject of the next section.

\section{The Onsager-Callen theory}

The thermoelectric processes have been nicely derived by Onsager and Callen, on the framework of the Thermodynamics of dissipative system by the link between out of equilibrium coupled processes (Onsager 1931a), (Onsager 1931b) and (Callen 1948). This description is based on the assumption that the system evolution is driven by a minimal production of entropy where each fluctuation of any thermodynamical potential undergoes a restoring force to equilibrium (Rocard 1967). This leads to a stationary picture where all the thermodynamical potential can be defined, though the system itself produces dissipation. It should be point out here that this description is also a definition of a quasi-static process because the different intensive parameters of the system are defined at each time and location. This implies that the intrinsic local time constants are much shorter than the time evolution of the macroscopic system itself. As a consequence, the classical quasi-static relation $d S=\frac{d Q_{q s}}{T}$ between the heat and the entropy variation can be extended in a flux form expression,

$$
\vec{J}_{S}=\frac{\vec{J}_{Q}}{T}
$$

The quasi-static conditions, previously defined, leads to a continuous thermodynamical description where the thermodynamical equilibrium, with all average fluxes equal zero, just becomes one possible thermodynamical state for the system.

\subsection{Forces and fluxes}

Let us consider the complete energy flux. From first principle of thermodynamics the energy flux $\vec{J}_{E}$, heat flux $\vec{J}_{Q}$ and the particles flux $\vec{J}_{N}$ are link through the conservation of the energy expression,

$$
\vec{J}_{E}=\vec{J}_{Q}+\mu_{e} \vec{J}_{N}
$$

Moreover each of these fluxes is conjugated to its thermodynamics potential gradients, acting as a generalized force. According to energy and particle conservation, in the case of a Fermion gas the correct potentials for energy and particles are $\frac{1}{T}$ and $\frac{\mu_{e}}{T}$.Then the corresponding forces are:

$$
\begin{gathered}
\vec{F}_{N}=\vec{\nabla}\left(-\frac{\mu_{e}}{T}\right) \\
\vec{F}_{E}=\vec{\nabla}\left(\frac{1}{T}\right)
\end{gathered}
$$

If we now write down the linear coupling of forces and fluxes we get then the transport of energy and particles is given by a linear set of coupled equation (Pottier 2007),

$$
\left[\begin{array}{c}
\vec{J}_{N} \\
\vec{J}_{E}
\end{array}\right]=\left[\begin{array}{ll}
L_{N N} & L_{N E} \\
L_{E N} & L_{E E}
\end{array}\right]\left[\begin{array}{c}
\vec{\nabla}\left(-\frac{\mu_{e}}{T}\right) \\
\vec{\nabla}\left(\frac{1}{T}\right)
\end{array}\right]
$$


with,

$$
L_{N E}=L_{E N}
$$

This expression of symmetry inside the out of equilibrium process is fundamental in the Onsager description since it is equivalent to the assumption of a minimal entropy production of the system under out of equilibrium conditions. It should be noticed that the minimal entropy production is not a general property of out of equilibrium processes at all and this imposes a precise delimitation of the validity of the model. The Onsager description gives very strong arguments in favor of this assumption. First, from the linear response theory it is known that linear response and fluctuations inside a dissipative system are closely linked. In the present case this implies that every fluctuating potential undergoes a restoring force from the other in a symmetric form. From a purely thermodynamic point of view this coincides with the Lechatelier-Braun principle. Then the $L_{N E}=L_{E N}$ equality traduces the intrinsic symmetry of the coupled fluctuations process. From a microscopic point of view this equality also implies the time reversal symmetry of the processes at micro scale, which should be "micro-reversible".

\subsection{Energy flux, heat flux}

For sake of simplicity it is more convenient to use the heat fluw $\vec{J}_{\mathrm{Q}}$ instead of the energy flux $\vec{J}_{E}$. Then, using [2] we get,

$$
\begin{gathered}
{\left[\begin{array}{c}
\vec{J}_{N} \\
\vec{J}_{Q}
\end{array}\right]=\left[\begin{array}{cc}
L_{11} & L_{12} \\
L_{21} & L_{22}
\end{array}\right]\left[\begin{array}{c}
-\frac{1}{T} \vec{\nabla}\left(\mu_{e}\right) \\
\frac{\vec{\nabla}\left(\frac{1}{T}\right)}{T}
\end{array}\right]} \\
L_{12}=L_{21}
\end{gathered}
$$

with the kinetic coefficients equivalence.

$$
\begin{gathered}
L_{11}=L_{N N} \\
L_{12}=L_{N E}-\mu_{e} L_{N N} \\
L_{22}=L_{E E}-\mu_{e}\left(L_{E N}+L_{N E}\right)+\mu_{e} L_{N N}
\end{gathered}
$$

\subsection{Basic transport results}

From the two expressions of particle and heat flux we can now derive the expressions of uncoupled and coupled transport processes, depending on the working conditions, isothermal, adiabatic, open or close systems.

\subsubsection{Ohm's law}

If considering charged particles we then get the expression of the current density,

$$
\vec{J}=-e L_{11} \frac{1}{T} \vec{\nabla}\left(\mu_{e}\right)
$$

Under isothermal conditions we can directly consider the particle flux in a simplified form,

$$
\vec{J}=e \vec{J}_{N}
$$

Since the electric field derives from the electrochemical potential we obtain, 


$$
\vec{E}=-\frac{\vec{\nabla}\left(\mu_{e}\right)}{e}
$$

which finally gives the expression of the isothermal electrical conductivity,

$$
\sigma_{T}=\frac{e^{2}}{T} L_{11}=\frac{e^{2}}{T} L_{N N}
$$

then,

$$
L_{11}=\frac{T}{e^{2}} \sigma_{T}
$$

\subsubsection{Fourier law}

In order to estimate the thermal conductivity we now consider the heat flux density in the absence of any particle transport, or under zero electrical current in the case of charged particles.

$$
\overrightarrow{J_{N}}=\overrightarrow{0}=-L_{11}\left(\frac{1}{T} \vec{\nabla}\left(\mu_{e}\right)\right)+L_{12} \vec{\nabla}\left(\frac{1}{T}\right)
$$

then,

$$
\left(\frac{1}{T} \vec{\nabla}\left(\mu_{e}\right)\right)=\frac{L_{12}}{L_{11}} \vec{\nabla}\left(\frac{1}{T}\right)
$$

The heat flux density becomes,

$$
\vec{J}_{Q_{J}}=\frac{1}{T^{2}}\left[\frac{L_{21} L_{12}-L_{11} L_{22}}{L_{11}}\right] \vec{\nabla}(T)
$$

and finally the thermal conductivity under zero electrical current is,

$$
\kappa_{J}=\frac{1}{T^{2}}\left[\frac{L_{11} L_{22}-L_{21} L_{12}}{L_{11}}\right]
$$

We can also consider the thermal conductivity under zero electrochemical gradient, $\kappa_{E}$, and after calculation we get,

$$
\vec{J}_{Q_{E}}=\frac{L_{22}}{T^{2}} \vec{\nabla}(T)=\kappa_{E} \vec{\nabla}(T)
$$

then

$$
\kappa_{E}=\frac{L_{22}}{T^{2}}
$$

\subsubsection{Seebeck coefficient}

We now consider the interaction between thermal and electrochemical processes, in the absence of particle transport. The basic expression is already known since it is given by equation [15]. We define the Seebeck coefficient as the ratio between the two forces, electrochemical gradient and temperature gradient, then the Seebeck coefficient expression is given by,

$$
\alpha \equiv-\frac{1}{e} \frac{\vec{\nabla}\left(\mu_{e}\right)}{\vec{\nabla}(T)}
$$




$$
\alpha=\frac{1}{e T} \frac{L_{12}}{L_{11}}
$$

\subsubsection{Peltier coefficient}

If we consider an isothermal configuration we can derive the expression of the coupling term between current density and heat flux, usually called the Peltier coefficient. Since

$$
\begin{aligned}
& \vec{J}=e L_{11}\left(-\frac{1}{T} \vec{\nabla}\left(\mu_{e}\right)\right) \\
& \vec{J}_{Q}=L_{21}\left(-\frac{1}{T} \vec{\nabla}\left(\mu_{e}\right)\right)
\end{aligned}
$$

then,

$$
\vec{J}_{Q}=\frac{1}{e} \frac{L_{12}}{L_{11}} \vec{J}
$$

The Peltier coefficient $\Pi$ is then given by,

$$
\begin{aligned}
& \vec{J}_{Q}=\Pi \vec{J} \\
& \Pi=\frac{1}{e} \frac{L_{12}}{L_{11}}
\end{aligned}
$$

As one can see we have the equality,

$$
\Pi=T \alpha
$$

This latter expression reveal the close connexion between Peltier and Seebeck effects. We will see latter that another effect, called Thomson effect is also strongly linked with theses two effect. The reason is that all of these effect depends on a quantity called the "'entropy per carrier"' which is now presented.

\subsubsection{Entropy per carrier}

If we get back to the first description of a thermoelectric cell as a Carnot like process, it is clear that the carriers inside the thermodynamic cycle transport a certain amount of entropy. Let us consider this by writing the entropy flux density,

$$
\vec{J}_{S}=\frac{\overrightarrow{J_{Q}}}{T}=\frac{1}{T}\left[L_{21}\left(-\frac{1}{T} \vec{\nabla}\left(\mu_{e}\right)\right)+L_{22} \vec{\nabla}\left(\frac{1}{T}\right)\right]
$$

This expression can be simplified considering the Ohm law expression [10]

$$
\vec{\nabla}\left(\mu_{e}\right)=-\vec{J} \frac{T}{e L_{11}}
$$

Then,

$$
\vec{J}_{S}=\frac{L_{21}}{T e L_{11}} \vec{J}+\frac{1}{T} L_{22} \nabla\left(\frac{1}{T}\right)
$$

As we can observe the entropy flux is driven by two terms coming from thermal and electrochemical contributions. The latter term shows that a fraction of the entropy is transported by the flux of carriers. We then get the expression of the "'entropy per carrier"', 


$$
S_{J}=\frac{L_{21}}{T L_{11}}
$$

As one can see the Seebeck coefficient is directly proportional to $S_{J}$ since we have,

$$
S_{J}=e \alpha
$$

The entropy per particle is a fundamental parameter since all the thermoelectric effects derive from its value, Seebeck effect, or its variations Peltier and Thomson effects.

\subsubsection{Kinetic coefficients and transport parameters}

From the previous results we have now the complete expressions of the kinetic coefficients,

$$
\begin{aligned}
& L_{11}=\frac{T}{e^{2}} \sigma_{T} \\
& L_{12}=\frac{T^{2}}{e^{2}} \sigma_{T} S_{J} \\
& L_{22}=\frac{T^{3}}{e^{2}} \sigma_{T} S_{J}^{2}+T^{2} \kappa_{J}
\end{aligned}
$$

The thermal conductivities under zero electrochemical gradient or zero particle transport can then be expressed:

$$
\kappa_{E}=T \alpha^{2} \sigma_{T}+\kappa_{J}
$$

We see that $\kappa_{J}$ is a purely conductive contribution meanwhile $\kappa_{E}$ contains both conductive and convective terms.

\section{Heat and entropy}

\subsection{Heat flux}

Let us consider again the coupled Onsager expressions:

$$
\begin{aligned}
\vec{J} & =-\sigma_{T}\left(\frac{\vec{\nabla}\left(\mu_{e}\right)}{e}\right)+\frac{\sigma_{T} S_{J} T^{2}}{e^{2}}\left(\vec{\nabla}\left(\frac{1}{T}\right)\right) \\
\vec{J}_{Q} & =-T \sigma_{T} S_{J}\left(\frac{\vec{\nabla}\left(\mu_{e}\right)}{e}\right)+\left[\frac{T^{3}}{e^{2}} \sigma_{T} S_{J}^{2}+T^{2} \kappa_{J}\right]\left(\vec{\nabla}\left(\frac{1}{T}\right)\right)
\end{aligned}
$$

Since the heat flux $\vec{J}_{Q}$ contains both conduction and convection contribution to heat flux. We can rewrite these equations in the form,

$$
\vec{J}_{Q}=T S_{J} \vec{J}+T^{2} \kappa_{J}\left(\vec{\nabla}\left(\frac{1}{T}\right)\right)
$$

where we identify a conductive and a convective contribution,

$$
\begin{aligned}
& \vec{J}_{Q_{\text {cond }}}=T^{2} \kappa_{J}\left(\vec{\nabla}\left(\frac{1}{T}\right)\right) \\
& \vec{J}_{Q_{\text {conv }}}=T S_{J} \vec{J}
\end{aligned}
$$




\subsection{Volumic heat production}

The volumic heat production can be estimated from the total energy flux,

$$
\vec{J}_{E}=\vec{J}_{Q}+\mu_{e} \vec{J}_{N}
$$

According to energy and particle conservation we have

$$
\begin{aligned}
& \vec{\nabla} \cdot \vec{J}_{E}=0 \\
& \vec{\nabla} \cdot \vec{J}_{N}=0
\end{aligned}
$$

Then,

$$
\vec{\nabla} \cdot \vec{J}_{Q}=-\vec{\nabla}_{\mu} \cdot \vec{J}_{N}
$$

or in an equivalent form,

$$
\vec{\nabla} \cdot \vec{J}_{Q}=\vec{E} \cdot \vec{J}
$$

This summarizes the possible transformation of the energy since it shows that heat can be produced by the degradation of the electrochemical potential $\mu_{e}$, and electrical power can be extracted from heat.

\subsection{Entropy production density}

If we consider the entropy flux density we can calculate the entropy production from,

$$
\vec{\nabla} \cdot \vec{J}_{S}=\stackrel{\circ}{S}=\vec{\nabla}\left(\frac{\vec{J}_{Q}}{T}\right)=\vec{\nabla}\left(\frac{1}{T}\right) \cdot \vec{J}_{Q}+\frac{1}{T} \vec{\nabla} \cdot \vec{J}_{Q}
$$

then,

$$
\stackrel{\circ}{V}=\vec{\nabla} \frac{1}{T} \cdot \vec{J}_{Q}-\frac{\vec{\nabla}_{\mu_{e}}}{T} \cdot \vec{J}_{N}
$$

A shown above, the entropy production is due to non isothermal heat transfers and electrical Joule production. This latter corresponds to a non adiabatic transfer of the entropy by the particles. The previous expression can be rewritten in the form,

$$
\stackrel{\circ}{S}=\vec{\nabla}\left(\frac{1}{T}\right) \cdot \vec{J}_{E}+\vec{\nabla}\left(-\frac{\mu_{e}}{T}\right) \cdot \vec{J}_{N}
$$

In this form, we get the illustration of one major result of the Onsager description:

The total entropy production is given by the summation of the force-flux products,

$$
\vec{\nabla} \cdot \vec{J}_{S}=\stackrel{\circ}{=}=\Sigma \overrightarrow{\text { force }} \cdot \overrightarrow{f l u x}
$$

This general result can also be obtained from minimal entropy production assumptions. 


\section{General conductivity matrix}

\subsection{Presentation}

As we have seen from Ohm law and Fourier law, the kinetic coefficients can be written in the form of conductances and it is tempting to express the force-flux expressions in the form of a general conductance matrix. It should be notice that such description has been derived in very general way by Callen and Greene in 1952 (Callen 1952), (Greene 1952). The complete derivation is out of the scope of this presentation and we only consider here the basic derivation of a general conductivity. Starting from equations [6] and [33] we have,

$$
\left[\begin{array}{c}
\vec{J}_{N} \\
\vec{J}_{Q}
\end{array}\right]=\left[\begin{array}{cc}
\frac{T}{e^{2}} \sigma_{T} & \frac{T^{2}}{e^{2}} \sigma_{T} S_{J} \\
\frac{T^{2}}{e^{2}} \sigma_{T} S_{J} & T^{2} \kappa_{E}
\end{array}\right]\left[\begin{array}{c}
-\frac{1}{\vec{T}} \vec{\nabla}\left(\mu_{e}\right) \\
\vec{\nabla}\left(\frac{1}{T}\right)
\end{array}\right]
$$

Using

$$
\vec{E}=-\frac{\vec{\nabla}\left(\mu_{e}\right)}{e}
$$

and considering that at local state $\vec{\nabla}\left(\frac{1}{T}\right)=-\frac{1}{T^{2}} \vec{\nabla}(T)$ then we get,

$$
\left[\begin{array}{c}
\vec{J}_{\vec{J}_{Q}}
\end{array}\right]=\left[\begin{array}{cc}
\sigma_{T} & \alpha \sigma_{T} \\
T \alpha \sigma_{T} & \kappa_{E}
\end{array}\right]\left[\begin{array}{c}
\vec{E} \\
-\vec{\nabla}(T)
\end{array}\right]
$$

Then the electrical and heat fluxes are totally described through the general conductivity matrix,

$$
[\sigma]=\left[\begin{array}{cc}
\sigma_{T} & \alpha \sigma_{T} \\
T \alpha \sigma_{T} & \kappa_{E}
\end{array}\right]
$$

One can notice that the Seebeck coefficient appears clearly as the coupling term between the electrical and thermal processes. In the case where $\alpha=0$, the conductivity matrix reduces to a diagonal form $\left[\begin{array}{cc}\sigma_{T} & 0 \\ 0 & \kappa_{E}\end{array}\right]$ where Ohm law and Fourier law are then totally decoupled. In other words the Seebeck coefficient, or more precisely the entropy per carrier is the "tunning parameter" of the coupling between electrical and thermal fluxes.

\subsection{Heat transformation}

In the previous paragraphs we have consider the volumic heat transformation from the calculation of the divergence of the heat flux $\vec{\nabla} \cdot \vec{J} \mathrm{Q}$. We propose now to get more deeply into this expression by considering its different terms. First, by elimination of the electrical field $\vec{E}$ from the previous set of equations we get,

$$
\vec{J}_{Q}=\alpha T \vec{J}-\kappa_{J} \vec{\nabla} T
$$

Then, calculating the heat flux divergence,

$$
\vec{\nabla} \cdot \vec{J}_{Q}=\vec{\nabla} \cdot\left[\alpha T \vec{J}-\kappa_{J} \vec{\nabla} T\right]=\left[T \vec{J} \cdot \vec{\nabla} \alpha+\alpha \vec{\nabla} T \cdot \vec{J}+\alpha T \vec{\nabla} \cdot \vec{J}+\vec{\nabla} \cdot\left[\kappa_{J}(-\vec{\nabla} T)\right]\right]
$$

we find four terms which can be identified:

$-\alpha T \vec{\nabla} \cdot \vec{J}$ : equal zero due to particle conservation. 
$-T \vec{J} \cdot \vec{\nabla} \alpha:$ "Peltier-Thomson" term.

$-\vec{J} \cdot \alpha \vec{\nabla} T:=\vec{J} \cdot\left[\vec{E}-\frac{\vec{J}}{\sigma_{T}}\right]=\vec{J} \cdot \vec{E}-\frac{J^{2}}{\sigma_{T}}$ : Electrical work production and dissipation.

$-\vec{\nabla} \cdot\left[\kappa_{J}\left(-\vec{\nabla}^{T}\right)\right]:$ heat production from non isothermal transfer.

then,

$$
\vec{\nabla} \cdot \vec{J}_{Q}=T \vec{J} \cdot \vec{\nabla} \alpha+\vec{J} \cdot \vec{E}-\frac{J^{2}}{\sigma_{T}}-\vec{\nabla} \cdot\left[\kappa_{J} \vec{\nabla} T\right]
$$

Most of these terms are common, but less intuitive is the Peltier-Thomson term which is now considered.

\subsection{The Peltier-Thomson term}

As we will show, the $T \vec{J} \cdot \vec{\nabla} \alpha$ term contains both the Thomson contribution (local, temperature gradient effect), and the Peltier contribution (isothermal, spatial gradient effect). Using the equivalence $\Pi=\alpha T$ we have,

$$
T \vec{J} \cdot \vec{\nabla} \alpha=T \vec{J} \cdot \vec{\nabla} \frac{\Pi}{T}=T \vec{J} \cdot\left[\frac{1}{T} \vec{\nabla} \Pi-\frac{1}{T^{2}} \Pi \vec{\nabla} T\right]=\vec{J} \cdot[\vec{\nabla} \Pi-\alpha \vec{\nabla} T]
$$

Then the traditional separation of the Peltier and Thomson contribution is artificial since they both refer to the same physic of the gradient of the entropy per particle, temperature driven gradient or spatially driven gradient.The isothermal configuration leads to Peltier expression meanwhile a spatial gradient gives the Thomson result.

- Pure Peltier $(\nabla T=0)$ :

$$
\vec{J} \cdot[\vec{\nabla} \Pi-\alpha \vec{\nabla} T]=\vec{J} \cdot[\vec{\nabla} \Pi]
$$

- Pure Thomson $(\alpha=f(T))$ :

$$
=\vec{J} \cdot[\vec{\nabla} \Pi-\alpha \vec{\nabla} \mathrm{T}]=\vec{J} \cdot\left[\frac{d \Pi}{d T}-\alpha\right] \vec{\nabla} \mathrm{T}=\tau \vec{J} \cdot \vec{\nabla} \mathrm{T}
$$

with,

$$
\begin{gathered}
\vec{\nabla} \Pi=\frac{d \Pi}{d T} \vec{\nabla} T \\
\tau=\frac{d \Pi}{d T}-\alpha
\end{gathered}
$$

then the heat flux divergence takes the form,

$$
\vec{\nabla} \cdot \vec{J}_{Q}=\tau \vec{J} \cdot \vec{\nabla}^{T}+\vec{J} \cdot \vec{E}-\frac{J^{2}}{\sigma_{T}}-\vec{\nabla} \cdot\left[\kappa_{J} \vec{\nabla}^{T}\right]
$$

If we consider a configuration $\kappa_{J} \neq f(T)$ then it reduces to,

$$
\vec{\nabla} \cdot \vec{J}_{Q}=\tau \vec{J} \cdot \vec{\nabla} T+\vec{J} \cdot \vec{E}-\frac{J^{2}}{\sigma_{T}}-\kappa_{J} \nabla^{2} T
$$




\subsection{Energy balance}

Using the expression [42] $\vec{\nabla} \cdot \vec{J}_{Q}=\vec{E} \cdot \vec{J}$ the local energy balance can be expressed from expression [57] (Landau 1984):

$$
\vec{\nabla} \cdot \vec{J}_{Q}-\vec{E} \cdot \vec{J}=\kappa_{T} \nabla^{2} T+\frac{J^{2}}{\sigma_{T}}-\tau \vec{J} \cdot \vec{\nabla} T=0
$$

It should be notice that this derivation does not need any assumption concerning the behavior of the particle, in equilibrium or not. In the case of transient configuration the energy balance equation should be corrected using $\rho C_{p}$ where $C_{p}$ is the heat capacitance and $\rho$ the volumic mass.

$$
\kappa_{J} \nabla^{2} T+\frac{J^{2}}{\sigma_{T}}-\tau \vec{J} \cdot \vec{\nabla} T=\rho C_{p} \frac{\partial T}{\partial t}
$$

\subsection{Entropy flux, volumic entropy production}

Using the expression [49] for the heat flux $\vec{J}_{Q}=\alpha T \vec{J}-\kappa_{J} \vec{\nabla} T$ we can derive the expression of the entropy flux,

$$
\vec{J}_{S}=\frac{\vec{J}_{Q}}{T}=\alpha \vec{J}-\frac{\kappa_{J}}{T} \vec{\nabla} T
$$

The calculation of the volumic entropy production is then straightforward,

$$
\begin{gathered}
\vec{\nabla} \cdot \vec{J}_{S}=\vec{\nabla}\left(\frac{1}{T}\right) \cdot \vec{J}_{Q}+\frac{1}{T} \vec{\nabla} \cdot \\
\vec{J}_{Q}=\vec{\nabla}\left(\frac{1}{T}\right) \cdot\left[\alpha T \vec{J}-\kappa_{J} \vec{\nabla} T\right]+\frac{1}{T} \vec{E} \cdot \vec{J}
\end{gathered}
$$

but since $\vec{E}=\frac{\vec{I}}{\sigma_{T}}+\alpha \vec{\nabla} T$ then,

$$
\vec{\nabla} \vec{J}_{S}=-\kappa_{J} \vec{\nabla}(T) \cdot \vec{\nabla}\left(\frac{1}{T}\right)+\frac{1}{T} \frac{J^{2}}{\sigma_{T}}
$$

And we verify that $\vec{\nabla} \cdot \vec{J}_{S}$ is in agreement with the force-flux description since,

$$
\begin{aligned}
& \vec{\nabla} \cdot \vec{J}_{S}=-\kappa_{J} \vec{\nabla}(T) \cdot \vec{\nabla}\left(\frac{1}{T}\right)+\frac{1}{T} \frac{J^{2}}{\sigma_{T}} \\
& =\left[-\kappa_{J} \vec{\nabla}(T)+\alpha \vec{J} T\right] \cdot \vec{\nabla}\left(\frac{1}{T}\right)+\vec{J} \cdot\left(\frac{\vec{E}}{T}\right) \\
& =\vec{J}_{Q} \cdot \vec{\nabla}\left(\frac{1}{T}\right)-\vec{J} \cdot \frac{\vec{\nabla} \tilde{\mu}_{e}}{e T}=\sum \text { flux.force }
\end{aligned}
$$

\subsection{Basic thermogenerator}

Let us consider a bar of thermoelectric material inserted between a hot and a cold sources respectively at temperatures $T_{h}$ and $T_{c}$, giving a very schematic picture of thermogenerator. As we know, electrical power can be extracted from the heat going through the bar. In such a device only a fraction of of the heat going trough is converted into electricital energy, and the maximum fraction is given by the Carnot efficiency 


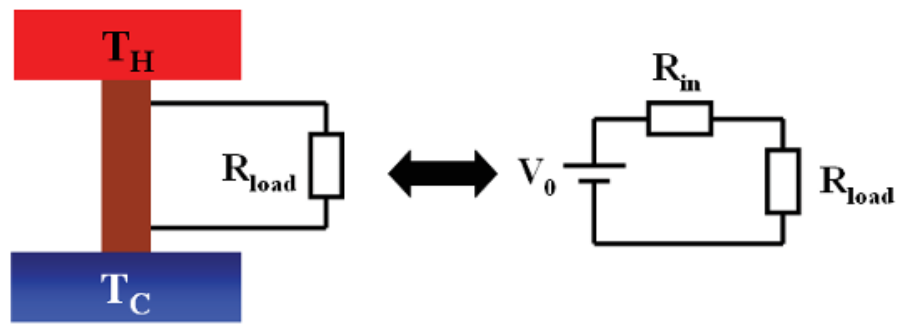

Fig. 2. Basic Thermogenerator.

$$
\eta_{C}=\frac{T_{h}-T_{C}}{T_{h}}
$$

Since the "thermoelectric engine" is not endoreversible the final fraction will be less than $\eta_{C}$. From the electrical point of view the system is equivalent to a voltage generator of open voltage $V_{0}$, internal resistance $R_{i n}$ and short-circuit current $I_{\mathcal{~ C C}}=\frac{V_{0}}{R_{i n}}$. The maximal electrical output power is obtained for $R_{\text {load }}=R_{\text {in }}$ giving

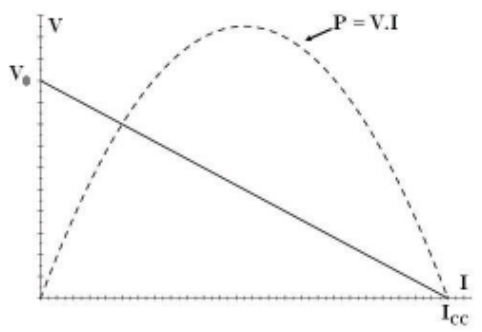

Fig. 3. IV Characteristic.

$$
P_{\max }=\frac{V_{0}^{2}}{4 R_{\text {in }}}=\frac{R_{\text {in }} I_{\mathcal{C C}}^{2}}{4}
$$

\subsection{Figure of merit}

As we have seen, if we force a heat flux to go through the thermoelectric material then an electrical current will flow. The efficiency of this process depends on two contributions:

- The material properties.

- The working conditions.

We will first consider the material properties, working conditions will be consider in a latter section.

The thermoelectric properties of a material are summarized in the so called "figure of merit" of a thermoelectric material, usually written "ZT". This scalar gives a direct measurement of the quality of the material, for practical applications. The ZT expression can be derived from technical arguments or from thermodynamical arguments. We propose here to derive the ZT expression from the thermal conductivity expressions. The illustration will be given on the basis of a thermogenerator description, but same conclusions could be drawn considering a Peltier heat pump configuration. 
Let us consider the schematic thermoelectric engine described in the introduction. From the electrical point of view we can consider to extreme cases, open circuit and short-circuit.

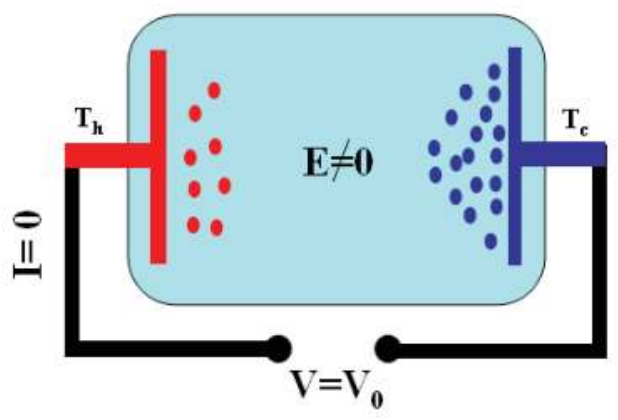

Fig. 4. Open circuit configuration.

These two configurations have strong consequences in term of thermal conductivity since it exists both an open circuit thermal conductivity $\kappa_{J}$ and a short-circuit thermal conductivity $\kappa_{E}$. These two are not independent since from [47] we have, $\kappa_{E}=T \alpha^{2} \sigma_{T}+\kappa_{J}$

Again we see that the coupling parameter between electrical and thermal processes is $\alpha$.

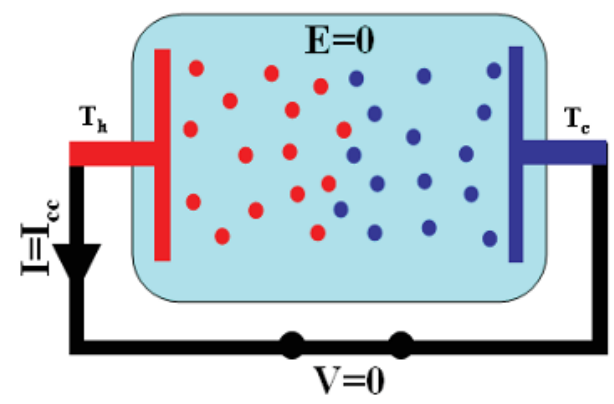

Fig. 5. Short circuit configuration.

From [33] we know that in order to get an efficient thermogenerator the open voltage should be as large as possible. As a consequence $\kappa_{J}$ should be minimal.

From the IV curve we can notice that, for a given open voltage, the larger is the short-circuit current, the larger will be the output current. In other word the ratio of the two thermal conductivities $\frac{\kappa_{E}}{\kappa_{I}}$ should be as large as possible. Then we can write,

$$
\frac{\kappa_{E}}{\kappa_{J}}=\left[\frac{\alpha^{2} \sigma_{T}}{\kappa_{J}} T+1\right]
$$

With

$$
\mathrm{ZT}=\frac{\alpha^{2} \sigma_{T}}{\kappa_{J}} T
$$


The maximal efficiency, and output electrical power, can be obtained if ZT is large enough. Since only material parameters enter into ZT expression, the figure of merit is clearly the central term for material engineering research. In addition it should be noticed that the present description do not consider at all the anions of the cristal lattice of the thermoelectric material but only the electronic gas ${ }^{2}$. This is due to the Onsager description which follows the so called linear response theory where the lattice contribution is not included. So the present description reduces to the electronic gas with no contribution of the vibrations of the cristal lattice (phonons) of the thermoelectric material. Such a lattice contribution may be added to the $L_{22}$ term as a lattice thermal conductivity $\kappa_{\text {Lat }}$. This latter can be added to the general conductance matrix by considering a parallel path for the heat flux, $\left[\begin{array}{cc}0 & 0 \\ 0 & \kappa_{\text {Lat }}\end{array}\right]$. The complete matrix then takes the form, $\left[\begin{array}{cc}\sigma_{T} & \alpha \sigma_{T} \\ T \alpha \sigma_{T} & \kappa_{E}+\kappa_{\text {Lat }}\end{array}\right]$.

\subsection{Isothermal and adiabatic electrical conductivity}

As we have seen the electrical current density expression is given by,

$$
\vec{J}=e \vec{J}_{N}=\sigma_{T}(\vec{E}-\alpha \vec{\nabla} T)
$$

with the electrical field ${ }^{3} \vec{E}=-\frac{\vec{\nabla} \mu_{e}}{e}$. Let us now consider an adiabatic process instead of a isothermal one then the conductance matrix gives us the adiabatic electrical conductivity in the form

$$
\vec{J}_{\vec{J}_{Q}=\overrightarrow{0}}=\frac{\sigma_{T}}{1+Z T} \vec{E}=\sigma_{Q} \vec{E}
$$

Again we see that the figure of merit ZT appears to be a fundamental thermodynamical parameter in the core of the description of the thermoelectric process leading to,

$$
\frac{\sigma_{T}}{\sigma_{Q}}=1+Z T
$$

\section{The thermoelectric potential}

Until now we have not really take into account the working conditions point of view. As any working engine a thermoelectric device should be correctly driven in order to provide power in the best conditions. Then a precise control of the applied thermodynamic potentials is needed in order to get a correct use of the potentialities of the thermoelectric materials. Since the thermoelectric process implies the coupling of the heat and electrical fluxes, these two fluxes should both be driven optimally. This question has been addressed by Jeffrey Snyder in 2003, using the so called relative current (Snyder 2003).

\subsection{Relative current and thermoelectric potential}

The relative current density defined by the ratio of the electrical flux and the purely conductive fraction of the heat flux.

$$
u=-\frac{J}{\kappa_{J} \nabla T}
$$

\footnotetext{
${ }^{2}$ This would be the same as considering a steam engine without any boiling walls!

${ }^{3} \vec{E}=\frac{\vec{I}}{\sigma_{T}}+\alpha \vec{\nabla} T$ so open voltage gives, $\vec{E}_{J=0}=\alpha \vec{\nabla} T$.
} 
From [49] the heat flow becomes,

$$
\begin{gathered}
\vec{J}_{Q}=T \alpha \vec{J}+\frac{\vec{J}}{u}=\Phi \vec{J} \\
\Phi=\left[T \alpha+\frac{1}{u}\right]
\end{gathered}
$$

where $\Phi$ is called the "Thermoelectric Potential".

The heat and carrier fluxes are now directly connected by the thermoelectric potential . This expression is fundamental since it allows to deriving the principle results of the Thermodynamics of thermoelectricity directly from this. According to the previous definitions the volumic heat production becomes,

$$
\vec{\nabla}_{\vec{J}}=\vec{J} \cdot \vec{\nabla}\left[\mathrm{T} \alpha+\frac{1}{u}\right]=\vec{J} \cdot \vec{\nabla} \Phi
$$

Since the heat production $\vec{J} \cdot \vec{\nabla} \frac{1}{u}$ directly reduces the efficiency, it becomes now evident that the maximum efficiency coincides with the minimization of $\vec{\nabla} \frac{1}{u}$. This is currently obtained for a specific value $u=s$, where $s$ is called "compatibility" (Snyder 2003).

Considering the entropy flux we get, $\vec{J}_{S}=\frac{1}{T}\left[\alpha+\frac{1}{u}\right] \vec{J}=\frac{\Phi}{T} \vec{J}$. The expression of the volumic entropy production becomes

$$
\vec{\nabla} \cdot \vec{J}_{S}=\vec{J} \cdot \vec{\nabla}\left(\frac{\Phi}{T}\right)
$$

We get the correct Onsager formulation of the entropy production as the summation of the Flux-Force products which here reduces to a single product. For a given material the thermoelectric potential give a direct measurement of the total volumic heat and entropy production by the respective degradation of $\Phi$ and $\frac{\Phi}{T}$. In other words, $\Phi$ can be considered to be the free energy of the electronic gas in the out of equilibrium process.

\subsection{The irreversible factor}

Due to the finite value of the ZT figure of merit, the maximal efficiency of a thermogenerator is only a fraction of the Carnot efficiency. In other word the thermoelectric engine is not endoreversible because ZT is not infinite. As we previously said the irreversiblities are partly due to finite value of ZT, but they are also due to the thermodynamic working conditions that can degrade strongly the overall efficiency. This is the subject of the present paragraph.

\subsection{Thermoelectric potential and efficiency}

Let us calculate the relative efficiency of a Thermoelectric Generator (TEG)(Goupil 2009). This latter is defined as the ratio between the electrical energy production and the entering heat flux. Using [42] and [1] we get $\vec{E} \cdot \vec{J}=\vec{\nabla} \cdot \vec{J}_{Q}=T \vec{\nabla} \cdot \vec{J}_{S}+\vec{J}_{S} \cdot \vec{\nabla}_{T}$. Then we obtain the expression of the relative efficiency,

$$
\eta_{r}=\frac{E . J}{J_{S} \nabla T}=\frac{E . J}{E . J+T \nabla J_{S}}
$$

In the ideal case of a reversible process the entropy production is zero then the power production reduces to $\vec{E} \cdot \vec{J}=\vec{J} S \cdot \vec{\nabla} T$ then the relative efficiency is $\eta_{r}=1$ which means that 
the Carnot efficiency is reached. In an irreversible configuration the $T \vec{\nabla} \cdot \vec{J}_{S}$ term contributes to reduce the efficiency then,

$$
\eta_{r}=\frac{E_{\text {out }} \cdot J}{J_{S} \cdot \nabla T}=\frac{E_{\text {out }} \cdot J}{E_{\text {out }} \cdot J+T \nabla J_{S}}
$$

where $E_{\text {out }} . J$ is the output electrical power. Let us now consider the introduction of the thermoelectric potential $\Phi$ into the calculation of the relative efficiency.

$$
\eta_{r}=\frac{\vec{E} \cdot \vec{J}}{\vec{J}_{S} \cdot \vec{\nabla} T}=\frac{\vec{\nabla} \cdot \vec{J}_{Q}}{\frac{\vec{J}_{Q}}{T} \cdot \vec{\nabla}^{T}}=\frac{\nabla \Phi}{\nabla^{T}} \frac{T}{\Phi}
$$

we see that $\eta_{r}$ corresponds to the relative variation of the thermodynamical potential $\frac{\nabla \Phi}{\Phi}$ when changing the other potential $\frac{\nabla T}{T}$. This is coherent with a general definition of the efficiency of an out of equilibrium thermodynamical process as a coupled fluctuating system ${ }^{4}$. Using the expression of the thermoelectric potential $\Phi$ we can now rewrite the relative current in the form,

$$
u=-\frac{J}{\kappa_{J} \nabla T}=-\frac{Z}{\alpha^{2}} \frac{\nabla \Phi}{\nabla T}+\frac{Z}{\alpha}
$$

Introducing the expression of the relative efficiency we get,

$$
u=\frac{Z}{\alpha}\left[-\left(1+\frac{1}{\alpha T u}\right) \eta_{r}+1\right]
$$

The relative efficiency of the section of TEG becomes (Snyder 2003),

$$
\eta_{r}=\frac{\frac{u \alpha}{Z}\left(1-\frac{u \alpha}{Z}\right)}{\left(\frac{u \alpha}{Z}+\frac{1}{Z T}\right)}=\frac{1-\frac{\alpha}{Z(\Phi-T \alpha)}}{1+\frac{Z(\Phi-T \alpha)}{Z T \alpha}}
$$

This classical expression of the reduce efficiency presents a maximum for the compatibility value $u_{o p t}=s=\frac{\sqrt{1+Z T}-1}{\alpha T}$. In that case the reduce efficiency becomes,

$$
\eta_{r}=\frac{\sqrt{1+Z T}-1}{\sqrt{1+Z T}+1}=\frac{1}{2 \frac{\Phi_{o p t}}{\alpha T}-1}
$$

where the equivalent optimal potential is,

$$
\Phi_{\text {opt }}=\left[T \alpha+\frac{1}{s}\right]=T \alpha\left[\frac{\sqrt{1+Z T}}{\sqrt{1+Z T}-1}\right]
$$

We can now plot the expression $\eta_{r}=f\left(\frac{\Phi}{T}\right)$ for various ZT values. One can notice that this $\Phi$ dependence is sharper as ZT values increase. This is in agreement with the assumption that the proximity to reversibility implies a drastic control of the working contributions. This is obtained by a direct control of $\Phi=\Phi_{\text {opt }}$. As a consequence it becomes possible to control the maximum relative efficiency by keeping $\Phi$ to its optimal value $\Phi=\Phi_{\text {opt }}$. This approach extends the compatibility approach by adding a thermodynamical feedback by a direct measurement of the local entropy production $\vec{\nabla} \cdot \vec{J}_{S}$. The expression of the irreversible

\footnotetext{
${ }^{4}$ We recover here another illustration of the equality $L_{i j}=\iota_{j i}$
} 


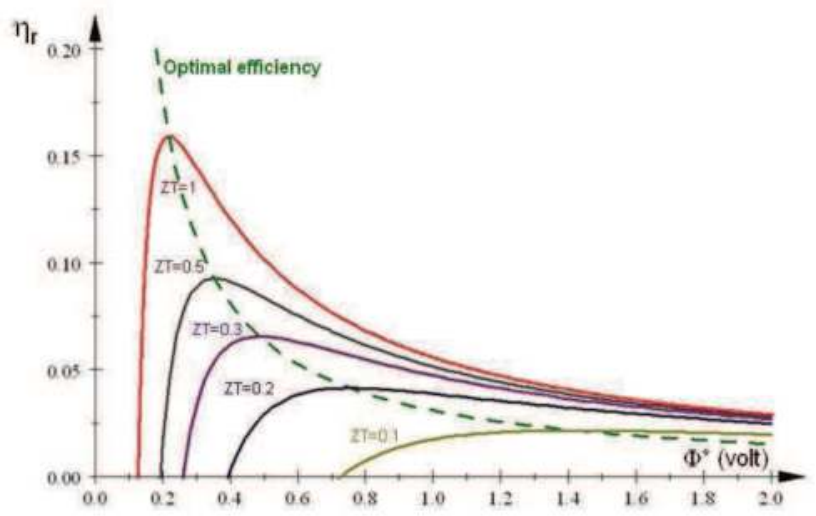

Fig. 6. Reduce Efficiency.

factor gives an insight of the sensibility of the thermoelectric system to varying working conditions. It shows that for large $\mathrm{ZT}$ this sensibility becomes very high and strong reduction of the efficiency can rapidly occur. For an optimal efficiency, obtained for $u=s$ it shows that $\Phi$ precises the intrinsic irreversibilities contribution for a given material.

\section{Conclusion}

The thermoelectric process has been described using a classical fluid approach. The "Fermi gas"' of electrons takes place of the traditional "steam" in the thermodynamical cycle, giving strong similarities in the description of the underlying mechanismes, which are based on the linear Onsager theory of "'out of equilibrium thermodynamics". It is shown that the so called "'figure of merit ZT"' of the thermoelectric material can be directly derived from this approach. Finally, the importance of the working conditions is demonstrated, leading to the concept of thermoelectric potential $\Phi$ which is an extension of the concept of free energy for the gas, under out of equilibrium conditions.

\section{References}

[Callen 1952] Herbert B. Callen and Richard F. Greene, Phys Rev 86 (5) 702-710 (1952).

[Callen 1954] Callen, H. B., Irreversible thermodynamics of thermoelectricity, Rev. Mod. Phys., 26, 237, 1954.

[Callen 1948] Herbert B. Callen, Phys Rev 73 (11) 1349-1358 (1948).

[Domenicali 1954] C.A. Domenicali, Rev. Mod. Phys. 26, 237-275, (1954).

[Goupil 2009] C. Goupil, J. Appl. Phys. 106, 104907 (2009). 5

[Greene 1952] Richard F. Greene and Herbert B. Callen, Phys Rev 88 (6) 1387-1391 (1952).

[Ioffe 1960] A.F. Ioffe, Physics of Semiconductors (Infosearch, London, 1960)

[Landau 1984] L. D. Landau and E. M. Lifshitz, Electrodynamics of Continuous Media, 2nd Edition, Butterworth Heinemann (Oxford, 1984).

[Onsager 1931a] L. Onsager, Phys. Rev. 37, 405 - 426 (1931).

[Onsager 1931b] L. Onsager, Phys. Rev. 38, 2265 - 2279 (1931).

[Peltier 1834] Peltier, J. C. A., Nouvelles experiences sur la caloricité des courants electrique, Ann. Chem. Phys., 56, 371, 1834. 
[Pottier 2007] Noëlle Pottier, Physique statisitique hors équilibre, processus irréversibles linéaires. Savoirs Actuels EDP Sciences/CNRS Editions, (2007).

[Rocard 1967] Y. Rocard ; Thermodynamique, Masson (2e édition-1967).

[Seebeck 1821] Seebeck, T. J., Ueber den magnetismus der galvenische kette, Abh. K. Akad. Wiss. Berlin, 289, 1821.

[Seebeck 1826] Seebeck, T. J., Ann. Phys. (Leipzig), 6, 1, 1826. 4. Seebeck, T. J., Methode, Platinatiegel auf ihr chemische reinheit durck thermomagnetismus zuprufen, Schweigger's J. Phys., 46, 101, 1826.

[Seebeck 1823] Seebeck, T. J., Magnetische polarisation der metalle und erze durck temperatur-differenz, Abh. K. Akad. Wiss. Berlin, 265, 1823.

[Snyder 2003] G. Jeffrey Snyder and Tristan S. Ursell,Phys. Rev. Lett. 91148301 (2003).

[Thompson 1848] Thomson, W., On an absolute thermometric scale, Philos. Mag., 33, 313, 1848

[Thompson 1849] Thomson, W., An account of Carnot's theory of the motive power of heat, Proc. R. Soc. Edinburgh, 16, 541, 1849.

[Thompson 1852] Thomson, W., On a mechanical theory of thermo-electric currents, Philos. Mag. [5], 3, 529, 1852.

[Thompson 1854] Thomson, W., Account of researches in thermo-electricity, Philos. Mag. [5], $8,62,1854$.

[Thompson 1856] Thomson, W., On the electrodynamic qualities of metals, Philos. Trans. R. Soc. London, 146, 649, 1856.

[Vinning 1997] C. B. Vining. Materials Research Society Symposium Proceedings: Thermoelectric Materials - Mater. Res. Soc. 278 (1997) 3-13. 


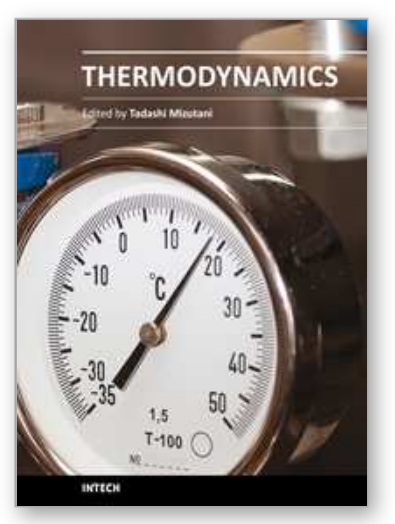

\author{
Thermodynamics \\ Edited by Prof. Mizutani Tadashi
}

ISBN 978-953-307-544-0

Hard cover, 440 pages

Publisher InTech

Published online 14, January, 2011

Published in print edition January, 2011

Progress of thermodynamics has been stimulated by the findings of a variety of fields of science and technology. The principles of thermodynamics are so general that the application is widespread to such fields as solid state physics, chemistry, biology, astronomical science, materials science, and chemical engineering. The contents of this book should be of help to many scientists and engineers.

\title{
How to reference
}

In order to correctly reference this scholarly work, feel free to copy and paste the following:

Christophe Goupil (2011). Thermodynamics of Thermoelectricity, Thermodynamics, Prof. Mizutani Tadashi (Ed.), ISBN: 978-953-307-544-0, InTech, Available from:

http://www.intechopen.com/books/thermodynamics/thermodynamics-of-thermoelectricity

\section{INTECH}

open science | open minds

\section{InTech Europe}

University Campus STeP Ri

Slavka Krautzeka 83/A

51000 Rijeka, Croatia

Phone: +385 (51) 770447

Fax: +385 (51) 686166

www.intechopen.com

\section{InTech China}

Unit 405, Office Block, Hotel Equatorial Shanghai

No.65, Yan An Road (West), Shanghai, 200040, China 中国上海市延安西路65号上海国际贵都大饭店办公楼 405 单元

Phone: +86-21-62489820

Fax: $+86-21-62489821$ 
(C) 2011 The Author(s). Licensee IntechOpen. This chapter is distributed under the terms of the Creative Commons Attribution-NonCommercialShareAlike-3.0 License, which permits use, distribution and reproduction for non-commercial purposes, provided the original is properly cited and derivative works building on this content are distributed under the same license. 